H. Yoshida

Nagoya Math. J.

Vol. 58 (1975), 69-82

\title{
SOME REMARKS ON ANGULAR RANGES AND SEQUENCES OF $\rho$-POINTS FOR HOLOMORPHIC FUNCTIONS
}

\author{
H. YOSHIDA
}

\section{Introduction}

In this paper, we will give examples of holomorphic functions in the unit disc having singular connections between the growth of maximum modulus and angular ranges (Theorem A) as well as singular connections between the growth of spherical derivative and sequences of $\rho$-points (Theorem B).

\section{Notation and preliminaries}

In the following, we denote the unit disc $\{z:|z|<1\}$ by $D$, the unit circle $\{z:|z|=1\}$ by $C$ and the finite $w$-plane by $W$.

Let $f(z)$ be holomorphic in $D$. The set of all values $w \in W$ such that the equation $f(z)=w$ has infinitely many solutions in a Stolz angle $\Delta(\zeta)$ having the vertex at $\zeta \in C$ is called the range of $f(z)$ in $\Delta(\zeta)$, and is denoted by $R_{\Delta(\zeta)}(f)$. The angular range $\Lambda(f, \zeta)$ of $f(z)$ at $\zeta \in C$ is defined to be

$$
\Lambda(f, \zeta)=\bigcap_{\Delta(\zeta)} R_{\Delta(\zeta)}(f),
$$

where the intersection is taken over all Stolz angle $\Delta(\zeta)$ having the vertex at $\zeta \in C$.

For a number $\varepsilon, 0<\varepsilon<1$, and a point $z^{\prime} \in D$, we shall denote by $D\left(z^{\prime}, \varepsilon\right)$ the open disc $\left\{z:\left|z-z^{\prime}\right|<\varepsilon\left(1-\left|z^{\prime}\right|\right)\right\}$ and shall denote by $D^{*}\left(z^{\prime}, \varepsilon\right)$ the open non-Euclidean disc with non-Euclidean center $z^{\prime}$ and nonEuclidean radius

$$
\frac{1}{2} \cdot \log \frac{1+\varepsilon}{1-\varepsilon}
$$

Let $f(z)$ be meromorphic in $D$. A sequence of points $\left\{z_{n}\right\}$ in $D$ is called

Received December 13, 1973. 
a sequence of $\rho$-points for $f(z)$ if there are two strictly decreasing sequences $\left\{L_{n}\right\},\left\{\varepsilon_{n}\right\}$ satisfying $L_{n} \rightarrow 0(n \rightarrow \infty), \varepsilon_{n} \rightarrow 0(n \rightarrow \infty)$ and there exists a sequence $\left\{D_{n}\right\}, D_{n}=D\left(z_{n}, \varepsilon_{n}\right)$, of open discs in $D$, having the following property: In each disc $D_{n}$, the function $f(z)$ assumes all values of the Riemann sphere with the possible exception of two sets of values $E_{n}$ and $F_{n}$ whose chordal diameters do not exceed $L_{n}$. In the following, because of the geometrical simplicity, we use this as a definition for a sequence of $\rho$-points.

Remark. This definition is equivalent to the usual definition of a sequence of $\rho$-points (e.g., see ([3], pp. 279)). This is evident from the fact: Let $z^{\prime}$ be a point in $D$ and $\varepsilon$ be a number satisfying $0<\varepsilon<\frac{1}{3}$. Then, we have

$$
D\left(z^{\prime}, \frac{1}{2} \varepsilon\right) \subset D^{*}\left(z^{\prime}, \varepsilon\right) \subset D\left(z^{\prime}, \sqrt{3 \varepsilon}\right) .
$$

Here, we shall give the proof of this fact.

Consider the linear transformation $z=\left(t+z^{\prime}\right) /\left(1+\overline{z^{\prime}} \cdot t\right)$ from $|t|<1$ to $|z|<1$. Then, since $D^{*}\left(z^{\prime}, \varepsilon\right)$ is the image of the set $\{t:|t|<\varepsilon\}$ by $z=$ $\left(t+z^{\prime}\right) /\left(1+\overline{z^{\prime}} \cdot t\right)$, any $z, z \in D^{*}\left(z^{\prime}, \varepsilon\right)$, satisfies the inequality

$$
\left|z-z^{\prime}\right|=\frac{1-\left|z^{\prime}\right|^{2}}{\left|1+\overline{z^{\prime}} \cdot t\right|}|t| \leqq\left(1-\left|z^{\prime}\right|\right) \frac{2 \cdot|t|}{1-|t|} \leqq \sqrt{3 \cdot|t|}\left(1-\left|z^{\prime}\right|\right)
$$

and

$$
\left|z-z^{\prime}\right| \geqq \frac{|t|}{1+\left|\overline{z^{\prime}}\right||t|}\left(1-\left|z^{\prime}\right|^{2}\right) \geqq \frac{|t|}{2}\left(1-\left|z^{\prime}\right|\right) .
$$

From these inequalities, (1.1) immediately follows.

In the following, we denote the maximum modulus of $f(z)$ by $M(r, f)$, $M(r, f)=\max _{|z|=r}|f(z)|$, and the spherical derivative of $f(z)$ by $f^{*}(z)$,

$$
f^{*}(z)=\frac{\left|f^{\prime}(z)\right|}{1+|f(z)|^{2}}
$$

\section{Statements of results}

\section{A. Maximum modulus and angular ranges}

THEOREM A. There exists a function $f(z)$ holomorphic in $D$, whose maximum modulus tends to infinity as slowly as one wishes, with the 
property: For every $\zeta \in C, \Lambda(f, \zeta)=W$.

This Theorem A is sharper than two results of Seidel ([7], Theorem 6 and Theorem 7) and a result of Yoshida ([8], Theorem 4). Further, from (3.4) in the below and Remark in section 1, we see that this Theorem $\mathrm{A}$ is sharper than two results of Lange ([6], Corollary V and Corollary VII).

Let $f(z)$ be holomorphic in $D$. By an angular Picard point of $f(z)$ we mean a point $\zeta \in C$ at which the set $W-\Lambda(f, \zeta)$ contains at most one value. Bagemihl ([1]) proved the existence of a holomorphic function $\gamma(z)$ in $D$ with the property: Almost every and nearly every point of $C$ is an angular Picard point of $\gamma(z)$. But, from this Theorem A, we obtain the sharper result: There exists a function $f(z)$, holomorphic in $D$, whose maximum modulus tends to infinity as slowly as one wishes, with the property: Every point of $C$ is an angular Picard point of $f(z)$.

\section{B. Spherical derivative and sequences of $\rho$-points}

Gauthier ([3], Theorem 3) proved the following property: A function $f(z)$ meromorphic in $D$ is normal if and only if $f(z)$ has no sequence of $\rho$-points. Hence, we can say: For a function $f(z)$ meromorphic in $D$, $(1-|z|) f^{*}(z)$ is bounded in $D$ if and only if $f(z)$ has no sequence of $\rho-$ points. In comparison with this fact, we ask the following question: How many sequences of $\rho$-points can appear by the unboundedness of $(1-|z|) f^{*}(z)$ ? The following Theorem B answers this question in a direction.

THEOREM B. There exists a function $f(z)$, holomorphic in $D$, such that $(1-|z|) f^{*}(z)$ tends to infinity as slowly as one wishes, with the property: For every $\zeta \in C$, every chord terminating at $\zeta$ contains $a$ sequence of $\rho$-points for $f(z)$.

\section{Proof of Theorem A}

The proof of this Theorem A bases on the following Lemma 1 and Lemma 2 which will be proved later.

LEMMA 1. Let $n_{k}(k=1,2,3, \ldots)$ be an increasing sequence of positive integers satisfying

$$
n_{k+1} \geqq(2 k-1)^{2} n_{k} \quad(k=1,2,3, \cdots), \quad n_{2}>n_{1}>1 .
$$


Then, the function

$$
f(z)=\prod_{j=1}^{\infty}\left\{1-\left(\frac{z}{1-\left(1 / n_{j}\right)}\right)^{(2 j-1) n_{j}}\right\}
$$

is holomorphic in $D$ and has the property: For every $\zeta \in C, \Lambda(f, \zeta)=W$.

LEMMA 2. Let $\mu(r)$ be any real, positive, increasing function defined in $0 \leqq r<1$, such that

$$
\lim _{r \rightarrow 1} \mu(r)=\infty
$$

Let $n_{k}(k=1,2,3, \cdots)$ be an increasing sequence of positive integers satisfying

$$
\frac{4 \cdot n_{k-2} \cdot n_{k-1}}{n_{k-2}+n_{k-1}} \leqq n_{k} \quad(k=3,4,5, \cdots), \quad n_{2}>n_{1}>1,
$$

and

$$
\mu\left(1-\frac{1}{n_{k}}\right) \geqq \exp \left(\frac{1}{960}+4^{2 k+4}\right)
$$

Then, the function

$$
f(z)=\prod_{j=1}^{\infty}\left\{1-\left(\frac{z}{1-\left(1 / n_{j}\right)}\right)^{(2 j-1) n_{j}}\right\}
$$

is holomorphic in $D$ and has the property: For every $r, 1-\left(1 / n_{2}\right) \leqq r<1$,

$$
M(r, f) \leqq \mu(r)
$$

Proof of Theorem $A$. For any real, positive, increasing function $\mu(r), \lim _{r \rightarrow 1} \mu(r)=\infty$, defined in $0 \leqq r<1$, choose an increasing sequence $\left\{n_{k}\right\}$ of positive integers satisfying $(\alpha)$ in Lemma 1 and $(\beta),(\gamma)$ in Lemma 2. Then, we see from Lemma 1 and Lemma 2 that the function for this sequence $\left\{n_{k}\right\}$

$$
f(z)=\prod_{j=1}^{\infty}\left\{1-\left(\frac{z}{1-\left(1 / n_{j}\right)}\right)^{(2 j-1) n_{j}}\right\}
$$

has the required properties.

Proof of Lemma 1. This infinite product (3.1) converges absolutely and uniformly in every disc $\{z:|z| \leqq r<1\}$, and hence $f(z)$ is holomorphic in $D$. 
The zeros of $f(z)$ are

$$
\begin{aligned}
z_{k, \nu}= & \left(1-\frac{1}{n_{k}}\right) \cdot \exp \left[i \frac{2 \pi \nu}{(2 k-1) n_{k}}\right] \\
& \left(k=1,2,3, \cdots ; \nu=0,1,2, \cdots,(2 k-1) n_{k}-1\right) .
\end{aligned}
$$

We denote the set $\left\{z_{k, \nu}\right\}\left(\nu=0,1,2, \cdots,(2 k-1) n_{k}-1\right)$ by $Z_{k}$. We set

$$
\Gamma_{k, \nu}=\left\{z:\left|z-z_{k, \nu}\right|<1 /(2 k-1)^{3} n_{k}\right\}
$$

and denote by $G$ the set

$$
D-\bigcup_{k=1}^{\infty} \bigcup_{\nu=0}^{(2 k-1) n_{k}-1} \Gamma_{k, \nu} .
$$

Now, we need the following Lemma 3 which will be verified later.

LEMMA 3. Let $\left\{\varepsilon_{j}\right\}$ be a sequence of positive numbers satisfying $\lim _{j \rightarrow \infty} \varepsilon_{j}=0$. Then, any sequence $\left\{z_{k}^{\prime}\right\}, z_{k}^{\prime} \in Z_{k}$, contains a subsequence $\left\{z_{k_{j}}^{\prime}\right\}$ of $\left\{z_{k}^{\prime}\right\}$ such that the image by $f(z)$ in (3.1) of the disc $D\left(z_{k_{j}}^{\prime}, \varepsilon_{j}\right)$ covers $W$ with the possible exception of a set $\left\{w \in W: \chi(w, \infty)<\varepsilon_{j}\right\}$, where $\chi(w, \infty)$ denotes the chordal distance between $w$ and $\infty$.

We continue the proof of Lemma 1 . Let $\zeta$ be any point on $C$. For any chord $\chi$ terminating at $\zeta$, we write

$$
z_{k}=\chi \cap\left\{z:|z|=1-\frac{1}{n_{k}}\right\}
$$

Then, for some sequence $\left\{z_{k}^{\prime}\right\}, z_{k}^{\prime} \in Z_{k}$, we have

$$
\frac{\left|z_{k}^{\prime}-z_{k}\right|}{1-\left|z_{k}\right|} \leqq\left(1-\frac{1}{n_{k}}\right) \frac{2 \pi}{(2 k-1)} .
$$

Here, we put

$$
\varepsilon_{j}=\left(1-\frac{1}{n_{j}}\right) \frac{2 \pi}{(2 j-1)} .
$$

Then, by Lemma 3 , for this $\left\{\varepsilon_{j}\right\}$ and this sequence $\left\{z_{k}^{\prime}\right\}$, we can choose a subsequence $\left\{z_{k_{j}}^{\prime}\right\}$ of the sequence $\left\{z_{k}^{\prime}\right\}$ such that the image by $f(z)$ in (3.1) of the disc $D\left(z_{k_{j}}^{\prime}, \varepsilon_{j}\right)$ covers $W$ with the possible exception of a set $\left\{w \in W: \chi(w, \infty)<\varepsilon_{j}\right\}$. Hence, if we note the fact

$$
D\left(z_{k_{j}}^{\prime}, \varepsilon_{j}\right) \subset D\left(z_{k_{j}}, 2 \cdot \varepsilon_{j}\right)
$$


we see that

the image by $f(z)$ in (3.1) of the disc $D\left(z_{k_{j}}, 2 \cdot \varepsilon_{j}\right)$ covers $W$ with the possible exception of a set $\left\{w \in W: \chi(w, \infty)<\varepsilon_{j}\right\}$.

(Here, we remark the following fact: This (3.3) shows that $\left\{z_{k_{j}}\right\}$ is a sequence of $\rho$-points for this function $f(z)$. Hence, we obtain the conclusion that

for every $\zeta \in C$, every chord terminating at $\zeta$ contains a sequence of $\rho$-points for $f(z)$ in (3.1).)

This fact (3.3) shows that for any Stolz angle $\Delta(\zeta)$ having the vertex at $\zeta$, meeting with $\chi, R_{\Delta(\zeta)}(f)=W$. Thus we conclude that for any Stolz angle $\Delta(\zeta)$ having the vertex at $\zeta, R_{\Delta(\zeta)}(f)=W$ and hence $\Lambda(f, \zeta)=W$.

Proof of Lemma 2. Let $r$ be an arbitrary number satisfying $1-\left(1 / n_{2}\right) \leqq r<1$. Put

$$
\rho_{k}=\frac{1}{2}\left(2-\frac{1}{n_{k}}-\frac{1}{n_{k+1}}\right)
$$

and choose $k$ so that

$$
\rho_{k} \leqq r<\rho_{k+1}
$$

Here, we need the following Lemma whose proof will be given later.

LEMMA 4. This function $f(z)$ in (3.2) has the property: For any $z,|z|=\rho_{k}(k \geqq 1)$,

$$
|f(z)| \leqq \exp \left(\frac{1}{960}+4^{2 k+2}\right)
$$

Now, from this Lemma 4 and $(\gamma)$, we have

$$
\begin{array}{r}
M(r, f) \leqq M\left(\rho_{k+1}\right) \leqq \exp \left(\frac{1}{960}+4^{2 k+4}\right) \leqq \mu\left(1-\frac{1}{n_{k}}\right) \leqq \mu\left(\rho_{k}\right) \leqq \mu(r) \\
\left(1-\frac{1}{n_{2}} \leqq r<1\right)
\end{array}
$$

Thus, we obtain the conclusion of Lemma 2 .

Proof of Lemma 3. First of all, by the method analogous to Bagemihl, Erdös and Seidel ([2], pp. 137-138), we shall prove a prelimi- 
nary fact:

$$
\lim _{\substack{z \rightarrow 1 \\ z \in G}} f(z)=\infty
$$

For an arbitrary integer $k \geqq 1$, consider the annulus

$$
R_{k}=\left\{z: 1-\frac{1}{n_{k}} \leqq|z|<1-\frac{1}{n_{k+1}}\right\}
$$

and decompose the product (3.1) into four subproducts $P_{i}(z)(i=1,2,3,4)$ composed, respectively, of the factors corresponding to $1 \leqq j \leqq k-1$, $j=k, j=k+1, j \geqq k+2$, so that

$$
f(z)=\prod_{j=1}^{4} P_{i}(z)
$$

Let $z_{0} \in R_{k} \cap G$. Now, we shall determine lower bounds for $P_{i}\left(z_{0}\right)(i=1$, $2,3,4)$.

First, we have

$$
\left|P_{1}\left(z_{0}\right)\right| \geqq \prod_{i=1}^{k-1}\left\{\left(\frac{1-1 / n_{k}}{1-1 / n_{j}}\right)^{(2 j-1) n_{j}}-1\right\}
$$

Here, from the inequality

$$
e<\left(1-\frac{1}{n_{j}}\right)^{-n_{j}} \leqq 4
$$

and $(\alpha)$, we obtain that for $k \geqq 5$

$$
\left(\frac{1-1 / n_{k}}{1-1 / n_{j}}\right)^{(2 j-1) n_{j}}>e^{2 j-1}\left(1-\frac{1}{n_{k}}\right)^{(2 k-3) n_{k-1}} \geqq e \cdot 4^{-(1 /(2 k-3))} \geqq 4^{-(1 / 7)} e>2 .
$$

Hence, we have for $k \geqq 5$

$$
\left|P_{1}\left(z_{0}\right)\right| \geqq\left(4^{-(1 / 7)} e-1\right)^{k-1} .
$$

Next, the function $\left|P_{2}\left(z_{0}\right)\right|$ in the set obtained by deleting the discs $\Gamma_{k, \nu}\left(\nu=0,1,2,3, \cdots,(2 k-1) n_{k}-1\right)$ from $D$, attains its minimum at some point $\eta$ on the circumference of one of these disc. Hence, we have

$$
\left|P_{2}\left(z_{0}\right)\right| \geqq\left|1-\left(\frac{\eta}{1-1 / n_{k}}\right)^{(2 k-1) n_{k}}\right|
$$

where $\eta$ is of the form 


$$
\eta=\left(1-\frac{1}{n_{k}}\right) \cdot \exp \left[i \frac{2 \pi \nu}{(2 k-1) n_{k}}\right]+\frac{1}{(2 k-1)^{3} n_{k}} e^{i \theta}
$$

Thus, it follows easily that

$$
\left|P_{2}\left(z_{0}\right)\right| \geqq \frac{c_{1}}{(2 k-1)^{2}},
$$

where $c_{1}$ is a positive constant.

By the similar argument, we have

$$
\left|P_{3}\left(z_{0}\right)\right| \geqq \frac{c_{2}}{(2 k+1)^{2}},
$$

where $c_{2}$ is a positive constant.

Finally, we have

$$
\left|P_{4}\left(z_{0}\right)\right| \geqq \prod_{j=k+2}^{\infty}\left\{1-\left(\frac{1-1 / n_{k+1}}{1-1 / n_{j}}\right)^{(2 j-1) n_{j}}\right\} .
$$

Here, from (3.8) and $(\alpha)$, we obtain that for $j \geqq k+2$ and $k \geqq 2$,

$$
\begin{aligned}
\left(\frac{1-1 / n_{k+1}}{1-1 / n_{j}}\right)^{(2 j-1) n_{j}} & \leqq 4^{2 j-1}\left(1-\frac{1}{n_{k+1}}\right)^{(2 j-1) n_{j}} \\
& \leqq 4^{(2 j-1)} \exp \left[-(2 k)^{1+2 j-k-1}\right] \\
& \leqq \exp \left[2(2 j-1)-(2 k)^{1+2 j-k-1}\right]<\frac{1}{2}
\end{aligned}
$$

and

$$
\begin{aligned}
\sum_{j=k+3}^{\infty} \exp \left[2(2 j-1)-(2 k)^{1+2 j-k-1}\right] & =e^{4 k+2} \sum_{j=k+3}^{\infty}\left(e^{-2 k}\right)^{(2 k) 2^{j-k-1}-2(j-k-1) / k} \\
& =e^{4 k+2} \sum_{j=2}^{\infty}\left(e^{-2 k}\right)^{(2 k)^{j}-(2 / k) j} \\
& \leqq e^{4 k+2} \sum_{j=2}^{\infty}\left(e^{-2 k}\right)^{j} \leqq 2 \cdot e^{2}
\end{aligned}
$$

Hence, from the inequality

$$
\log (1-x)>-2 x \quad\left(0<x<\frac{1}{2}\right),
$$

it follows that for $k \geqq 2$

$$
\left|P_{4}\left(z_{0}\right)\right| \geqq \exp \left\{-2 \sum_{j=k+2}^{\infty} \exp \left[2(2 j-1)-(2 k)^{1+2^{j-k-1}}\right]\right\} \geqq e^{-1-4 e^{2}}>0 .
$$


Thus, the relations (3.7), (3.9), (3.10), (3.11) and (3.12) yield

$$
\left|f\left(z_{0}\right)\right| \geqq c_{3} \cdot \frac{\left(4^{-(1 / 7)} e-1\right)^{k-1}}{(2 k-1)^{2}(2 k+1)^{2}} \quad(k \geqq 5),
$$

where $c_{3}$ is a positive constant, and this shows (3.6).

Now, we proceed to the proof of Lemma 3.

Since any circle $\left\{z:\left|z-z_{k, \nu}\right|=1 /(2 k-1)^{2} n_{k}\right\}$ lies in $G$ and $f(z)$ has a zero only at $z_{k, \nu}$ in the disc $\left\{z:\left|z-z_{k, \nu}\right|<1 /(2 k-1)^{2} n_{k}\right\}$, the preliminary fact (3.6) and Rouche's theorem (e.g., see ([5], pp. 254)) shows that any sequence $\left\{z_{k}^{\prime}\right\}, z_{k}^{\prime} \in Z_{k}$, contains a subsequence $\left\{z_{k_{j}}^{\prime}\right\}$ of $\left\{z_{k}^{\prime}\right\}$ such that the image by $f(z)$ of the disc $\left\{z:\left|z-z_{k_{j}}\right|<1 /\left(2 k_{j}-1\right)^{2} n_{k}\right\}$ covers $W$ with the possible exception of a set $\left\{w \in W: \chi(w, \infty)<\varepsilon_{j}\right\}$. Here, we can suppose that the subsequence $\left\{z_{k_{j}}^{\prime}\right\}$ is chosen so that

$$
\frac{1}{\left(2 k_{j}-1\right)^{2}}<\varepsilon_{j} \text {. }
$$

Hence, if we pay attention to the fact

$$
\left\{z:\left|z-z_{k_{j}}^{\prime}\right|<\frac{1}{\left(2 k_{j}-1\right)^{2} n_{k_{j}}}\right\} \subset D\left(z_{k_{j}}^{\prime}, \varepsilon_{j}\right),
$$

the image by $f(z)$ of the disc $D\left(z_{k_{j}}^{\prime}, \varepsilon_{j}\right)$ covers $W$ with the possible exception of a set $\left\{w \in W: \chi(w, \infty)<\varepsilon_{j}\right\}$. Thus, Lemma 3 is proved.

Proof of Lemma 4. Let $k$ be an arbitrary positive integer. We decompose the product (3.2) into two subproducts $I_{i}(z)(i=1,2)$ composed, respectively, of the factors corresponding to $1 \leqq j \leqq k+1, j \geqq k+2$, so that

$$
f(z)=\prod_{i=1}^{2} I_{i}(z)
$$

Let $z_{0}$ be a point satisfying $\left|z_{0}\right|=\rho_{k}$. We shall determine the upper bounds for $I_{i}\left(z_{0}\right)(i=1,2)$.

First of all, from (3.8) we have

$$
\begin{aligned}
\left|I_{1}\left(z_{0}\right)\right| & \leqq \prod_{i=1}^{k+1}\left\{1+\left(\frac{1}{1-\left(1 / n_{j}\right)}\right)^{(2 j-1) n_{j}}\right\} \\
& \leqq \prod_{j=1}^{k+1}\left\{1+4^{2 j-1}\right\} \leqq \exp \left(\sum_{j=1}^{k+1} 4^{2 j-1}\right) \leqq \exp \left(4^{2 k+2}\right)
\end{aligned}
$$

Next, we have 


$$
\left|I_{2}\left(z_{0}\right)\right| \leqq \prod_{k+2}^{\infty}\left\{1+\left(\frac{\rho_{k}}{1-\left(1 / n_{j}\right)}\right)^{(2 j-1) n_{j}}\right\}
$$

Here, since

$$
\begin{aligned}
\rho_{k}^{(2 j-1) n_{j}} & =\left(1-1 / \frac{2 \cdot n_{k} n_{k+1}}{n_{k+1}+n_{k}}\right)^{\left[-2 \cdot n_{k} n_{k+1} /\left(n_{k+1}+n_{k}\right)\right]\left[-\left(n_{k+1}+n_{k}\right)(2 j-1) n_{j} / 2 \cdot n_{k} n_{k+1}\right]} \\
& \leqq 4^{\left[-\left(n_{k+1}+n_{k}\right)(2 j-1) n_{j} / 2 \cdot n_{k} n_{k+1}\right]}
\end{aligned}
$$

by the fact analogous to (3.8), we have from (3.8) and ( $\beta$ )

$$
\begin{aligned}
\left(\rho_{k} / 1-\frac{1}{n_{j}}\right)^{(2 j-1) n_{j}} & \leqq 4^{-(2 j-1)\left\{\left[\left(n_{k+1}+n_{k}\right) n_{k+2}-2 \cdot n_{k} n_{k+1}\right] / 2 \cdot n_{k} n_{k+1}\right\}} \\
& \leqq 4^{-(2 j-1)} \quad(j \geqq k+2) .
\end{aligned}
$$

Hence, we have

$$
\left|I_{2}\left(z_{0}\right)\right| \leqq \prod_{k+2}^{\infty}\left\{1+4^{-(2 j-1)}\right\} \leqq \exp \left[\sum_{k+2}^{\infty} 4^{-(2 j-1)}\right] \exp \left(\frac{1}{960}\right) \quad(k \geqq 1)
$$

Thus, we obtain the conclusion of Lemma 4 from (3.13), (3.14) and (3.15).

\section{Proof of Theorem B}

The proof of this Theorem B bases on Lemma 1 which was already proved and the following Lemma 5 which will be verified at the end of this proof of Theorem B.

LEMMA 5. Let $\left\{n_{k}\right\}(k=1,2,3, \cdots)$ be an increasing sequence of odd integers satisfying $(\beta),(\iota)$ and $(\kappa)$, where

$$
\begin{gathered}
n_{k+1} \geqq n_{k}^{2} \quad(k=1,2,3, \cdots), \quad n_{1}>1, \\
n_{k} \geqq \sum_{j=1}^{k-1} 4^{2 j-1}(2 j-1) n_{j} \quad(k \geqq 2) .
\end{gathered}
$$

Then, the function

$$
f(z)=\prod_{j=1}^{\infty}\left\{1-\left(\frac{z}{1-\left(1 / n_{j}\right)}\right)^{(2 j-1) n_{j}}\right\}
$$

is holomorphic in $D$ and has the property: For any $z, 1-\left(1 / n_{1}\right) \leqq|z|<1$,

$$
(1-|z|) f^{*}(z) \leqq\left(5 \cdot e \cdot 4^{2 k}+c\right) \cdot \exp \left(\frac{1}{960}+4^{2 k+4}\right),
$$


where $k$ is determined in such a way that

$$
1-\frac{1}{n_{k}} \leqq|z|<1-\frac{1}{n_{k+1}}
$$

and $c$ is a positive constant.

Proof of Theorem $B$. For any real, positive, increasing function $\mu(r), \lim _{r \rightarrow 1} \mu(r)=\infty$, defined in $0 \leqq r<1$, choose an increasing sequence $\left\{n_{k}\right\}$ of positive, odd integers satisfying $(\alpha),(\beta),(\iota),(\kappa)$ and $(\lambda)$, where

$$
\mu\left(1-\frac{1}{n_{k}}\right) \geqq\left(5 \cdot e \cdot 4^{2 k}+c\right) \cdot \exp \left(\frac{1}{960}+4^{2 k+4}\right) .
$$

Then, we shall prove that the function

$$
\left.f(z)=\prod_{j=1}^{\infty}\left\{1-\frac{z}{1-\left(1 / n_{j}\right)}\right)^{(2 j-1) n_{j}}\right\}
$$

has the required properties.

First, since the sequence $\left\{n_{k}\right\}$ satisfies $(\alpha)$, we see from (3.4) that for every $\zeta \in C$, any chord terminating at $\zeta$ contains a sequence of $\rho$-points for $f(z)$.

Next, since the sequence $\left\{n_{k}\right\}$ also satisfies $(\beta),(\iota),(\kappa)$ and $(\lambda)$, we have from Lemma 5, ( $\lambda$ ) and (4.1) that for any value $z, 1-\left(1 / n_{1}\right) \leqq|z|<1$,

$$
(1-|z|) f^{*}(z) \leqq\left(5 \cdot e \cdot 4^{2 k}+c\right) \cdot \exp \left(\frac{1}{960}+4^{2 k+4}\right) \leqq \mu\left(1-\frac{1}{n_{k}}\right) \leqq \mu(|z|),
$$

where $k$ is chosen as shown in (4.1).

These facts prove Theorem $\mathrm{B}$.

Proof of Lemma 5. We shall prove this lemma by the method analogous to Gavrilov ([4], Theorem 3). We put

$$
g_{j}(z)=\prod_{i \neq j}^{\infty}\left\{1-\left(\frac{z}{1-\left(1 / n_{i}\right)}\right)^{(2 i-1) n_{i}}\right\}
$$

Consider the derivative

$$
f^{\prime}(z)=-\sum_{j=1}^{\infty} \frac{(2 j-1) n_{j}}{\left(1-1 / n_{j}\right)^{(2 j-1) n_{j}}} \cdot z^{(2 j-1) n_{j}-1} g_{j}(z)
$$

Then, from (3.8) we have 


$$
\begin{aligned}
& (1-|z|) f^{*}(z) \leqq(1-|z|)\left|f^{\prime}(z)\right| \\
& \quad \leqq \sum_{j=1}^{\infty}(1-|z|) \cdot 4^{(2 j-1)}(2 j-1) n_{j} \cdot|z|^{(2 j-1) n_{j}-1}\left[\prod_{i=1}^{\infty}\left\{1+\left(\frac{|z|}{1-\left(1 / n_{i}\right)}\right)^{(2 i-1) n_{i}}\right\}\right] .
\end{aligned}
$$

Here, from the fact that $n_{j}$ is an odd integer, we have

$$
(1-|z|) f^{*}(z) \leqq M(|z|, f) \cdot \sum_{j=1}^{\infty}(1-|z|) \cdot 4^{2 j-1}(2 j-1) n_{j} \cdot|z|^{(2 j-1) n_{j}-1} .
$$

Now, we shall estimate the sum in (4.2)

$$
I(z)=\sum_{j=1}^{\infty}(1-|z|) \cdot 4^{2 j-1}(2 j-1) n_{j} \cdot|z|^{(2 j-1) n_{j-1}} .
$$

Let $z, 1-\left(1 / n_{1}\right) \leqq|z|<1$, be given arbitrarily in $D$, and $k$ be chosen as shown in (4.1). We decompose $I(z)$ into three partial sums $I_{i}(z)$ $(i=1,2,3)$ composed, respectively, of the factors corresponding to $1 \leqq j \leqq k-1, k \leqq j \leqq k+1, j \geqq k+2$, so that

$$
I(z)=\sum_{i=1}^{3} I_{i}(z),
$$

and we shall determine the upper bounds for $I_{i}(z)(i=1,2,3)$.

First of all, from $(\kappa)$ we have

$$
\left|I_{1}(z)\right| \leqq \frac{1}{n_{k}} \cdot \sum_{j=1}^{k-1} 4^{2 j-1}(2 j-1) n_{j} \leqq 1 .
$$

Next, using the inequalities

$$
x<e^{-(1-x)} \text { for } 0<x<1 \text { and } x \cdot e^{-x}<1 \text { for } x>0 \text {, }
$$

we have

$$
\begin{aligned}
\left|I_{2}(z)\right| \leqq & e \cdot 4^{2 k-1}(1-|z|)(2 k-1) n_{k} \cdot \exp \left[-(1-|z|)(2 k-1) n_{k}\right] \\
& +e \cdot 4^{2 k+1}(1-|z|)(2 k+1) n_{k+1} \cdot \exp \left[-(1-|z|)(2 k+1) n_{k+1}\right] \\
\leqq & 5 \cdot e \cdot 4^{2 k}
\end{aligned}
$$

Next, using (4.1) and (4.5), we have

$$
\begin{aligned}
\left|I_{3}(z)\right| \leqq & \sum_{j=k+2}^{\infty} e^{2(2 j-1)}(2 j-1) n_{j} \cdot \exp \left[-(1-|z|)\left((2 j-1) n_{j}-1\right)\right] \\
\leqq & \sum_{j=k+2}^{\infty}\left[(2 j-1)\left(n_{j}-2 \cdot n_{k+1}\right)-1\right] \cdot \exp \left[-\frac{(2 j-1)\left(n_{j}-2 \cdot n_{k+1}\right)-1}{n_{k+1}}\right] \\
& \quad+\sum_{j=k+2}^{\infty}\left[(2 j-1) \cdot 2 \cdot n_{k+1}-1\right] \cdot \exp \left[-\frac{(2 j-1)\left(n_{j}-2 \cdot n_{k+1}\right)-1}{n_{k+1}}\right]
\end{aligned}
$$




$$
+2 \cdot \sum_{j=k+2}^{\infty} \exp \left[-\frac{(2 j-1)\left(n_{j}-2 \cdot n_{k+1}\right)-1}{n_{k+1}}\right] .
$$

Here, from (८), we obtain

$$
\left[(2 j-1)\left(n_{j}-2 \cdot n_{k+1}\right)-1\right]^{\frac{1}{2}} \geqq n_{k+1} \quad(j \geqq k+2)
$$

and

$$
n_{j}-2 \cdot n_{k+1} \geqq n_{k+2}-2 \cdot n_{k+1} \geqq 2 \cdot n_{k+1} \quad(j \geqq k+2) .
$$

Hence, we have

$$
\begin{aligned}
\left|I_{3}(z)\right| \leqq & \sum_{j=k+2}^{\infty}\left[(2 j-1)\left(n_{j}-2 \cdot n_{k+1}\right)-1\right] \\
& \cdot \exp \left[-\left\{(2 j-1)\left(n_{j}-2 \cdot n_{k+1}\right)-1\right\}^{\frac{1}{2}}\right] \\
& +\sum_{j=k+2}^{\infty}\left[(2 j-1) \cdot 2 \cdot n_{k+1}-1\right] \cdot \exp \left[-\left\{(2 j-1) \cdot 2 \cdot n_{k+1}-1\right\}^{\frac{1}{2}}\right] \\
& +2 \cdot \sum_{j=k+2}^{\infty} \exp \left[-\left\{(2 j-1)\left(n_{j}-2 \cdot n_{k+1}\right)-1\right\}^{\frac{1}{2}}\right] \\
\leqq & 2 \cdot \int_{0}^{\infty} x \cdot \exp \left(-x^{\frac{1}{2}}\right) \cdot d x+2 \cdot \int_{0}^{\infty} \exp \left(-x^{\frac{1}{2}}\right) \cdot d x=c^{\prime}
\end{aligned}
$$

where $c^{\prime}$ is a positive constant.

Thus, from (4.3), (4.4), (4.6) and (4.7), we obtain

$$
|I(z)| \leqq 1+5 \cdot e \cdot 4^{2 k}+c^{\prime}=5 \cdot e \cdot 4^{2 k}+c,
$$

where $c$ is a positive constant.

On the other hand, since $\left\{n_{j}\right\}$ satisfies $(\beta)$, we have from (3.5) and

$$
M(|z|, f) \leqq M\left(\rho_{k+1}, f\right) \leqq \exp \left(\frac{1}{960}+4^{2 k+4}\right)
$$

Thus, we obtain from (4.2), (4.8) and (4.9)

$$
(1-|z|) f^{*}(z) \leqq\left(5 \cdot e \cdot 4^{2 k}+c\right) \cdot \exp \left(\frac{1}{960}+4^{2 k+4}\right) .
$$

\section{REFERENCES}

[1] F. Bagemihl, Chordal limits of holomorphic functions at Plessner points, J. Sci. Hiroshima Univ. 30 (1966) 109-115.

[2] - P. Erdös, and W. Seidel, Quelques propriétés frontières des fonctions holomorphes définies par certains produits dans le cercle-unité, Ann. École Norm. Sup. 70 (1953) 135-147. 
[ 3 ] P. Gauthier, A criterion for normalcy, Nagoya Math. J. 32 (1968) 277-282.

[ 4 ] V. I. Gavrilov, On a uniqueness theorem, Nagoya Math. J. 35 (1969) 151-157.

[ 5 ] E. Hille, Analytic function theory, Vol. 1, (Blaisdell, 1962).

[ 6 ] L. H. Lange, Sur les cercles de remplissage non-euclidiens, Ann. École Norm. Sup. 77 (1960) 257-280.

[ 7 ] W. Seidel, Holomorphic functions with spiral asymptotic paths, Nagoya Math. J. 14 (1959) 159-171.

[ 8 ] H. Yoshida, On the boundary behavior of holomorphic functions in the unit disc, to appear.

\section{Chiba University}

